\title{
La importancia de los factores socioculturales en la competitividad de la empresa rural. El caso de la empresa apícola Miel Tierra Grande
}

The Importance of Sociocultural Factors in the Competitiveness of the Rural Company. The Case of the Apiarian Company Miel Tierra Grande

A importância dos fatores socioculturais na competitividade das empresas rurais. $O$ caso da empresa apícola Miel Tierra Grande

Indira Flores Salgado Universidad Autónoma de Guerrero, Centro de Investigación y Posgrado en Estudios Socioterritoriales, México Indy_088@gmail.com https://orcid.org/0000-0001-8815-4925

Floriberto González González Universidad Autónoma de Guerrero, Centro de Investigación y Posgrado en Estudios Socioterritoriales, México florgogo2014@gmail.com https://orcid.org/0000-0002-4964-0733 


\section{Revista Iberoamericana \\ de las Ciencias Sociales y \\ Humanísticas}

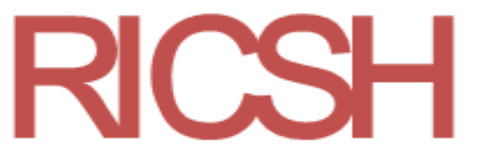

ISSN: 2395 - 7972

\section{Resumen}

La presente investigación analiza si los recursos de índole sociocultural que posee una empresa inserta en el medio rural tienen influencia en su competitividad y desarrollo. El objeto de estudio es una organización rural productiva denominada Miel Tierra Grande, localizada en la comunidad de Hueyitlalpan del estado de Guerrero. Su giro es la apicultura, dedicada a la producción de colmenas para la extracción de miel y sus derivados como polen, propóleo y jalea real, que son transformados para generar otros productos desde el ramo alimenticio hasta productos para el cuidado e higiene personal. Para analizar su competitividad, se realizaron entrevistas semiestructuradas; la primera a uno de los socios de la empresa encargado de la planta de producción; ocho entrevistas a habitantes de la localidad, y una al comisario. Además, se realizaron seis visitas para observar el proceso de producción, distribución y comercialización de los productos. En cuanto a los aspectos sociales y culturales que poseen las organizaciones rurales, se realizó una búsqueda de bibliohemerografía para asentar las bases teóricas. La empresa rural analizada posee un estilo particular de organización derivado de las características socioculturales de la población; se trata de una de las empresas más importantes de la región centro del estado. Se detectó que factores socioculturales como la religión, la relación con el entorno, la población, las relaciones familiares y el conocimiento local influyen en la construcción de una forma de organización en la empresa rural, todo lo cual la hacen capaz de ser competitiva. Se redefinió el concepto de competitividad aplicado a las empresas rurales; aquí se trata de la capacidad que tiene un sistema organizado de individuos para generar bienes o servicios de calidad a costos mínimos, que estén en posibilidad de mantenerse en el mercado (local, regional, nacional o extranjero), con la finalidad de lograr un beneficio económico y social.

Palabras clave: competitividad social, cultura, empresa rural, identidad, territorio rural. 


\section{Revista Iberoamericana \\ de las Ciencias Sociales y \\ Humanísticas}

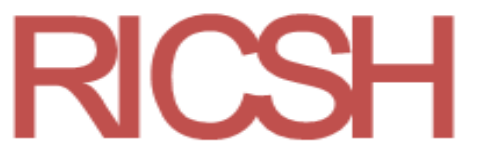

ISSN: $2395-7972$

\section{Abstract}

This research analyzes if the socio-cultural resources that a company has in rural areas have an influence on their competitiveness and development. The object of this study is a rural productive organization named Miel Tierra Grande, located in the community of Hueyitlalpan of the state of Guerrero, Mexico. Their main production is the beekeeping, dedicated to the production of hives for the extraction of honey and its derivatives such as pollen, propolis and royal jelly, which are transformed to generate other products, ranging from the food industry to personal care and hygiene products. To analyze their competitiveness, semi-structured interviews were conducted: the first to one of the partners of the company in charge of the production plant, eight interviews with locals and one with the commissioner. In addition, six visits were made to observe the process of production, distribution and marketing of the products; visits were made to outlets outside the town. A search for bibliohemerography related to the social and cultural aspects of rural organizations was carried out. The rural company analyzed has a style of organization derived from the sociocultural characteristics of the population; it is one of the most important companies in the central region of the state. Sociocultural factors such as religion, relationship with environment, population, family relations and local knowledge were found to influence the construction of a form of organization in the rural enterprise making it capable of being competitive. The concept of competitiveness applied to rural businesses was described as the ability of an organized system of individuals to generate quality goods and services, at minimum costs, that are able to remain in the market (local, regional, national or foreign), in order to achieve an economic and social benefit.

Keywords: social competitiveness, culture, rural enterprise, identity rural territory. 


\section{Revista Iberoamericana \\ de las Ciencias Sociales y \\ Humanísticas}

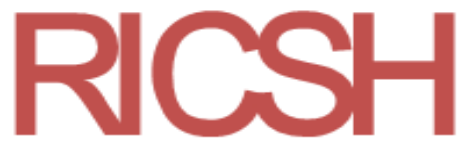

ISSN: $2395-7972$

\section{Resumo}

Esta pesquisa analisa se os recursos socioculturais que uma empresa localizada no meio rural possui influenciam sua competitividade e desenvolvimento. $\mathrm{O}$ objeto de estudo é uma organização rural produtiva denominada Miel Tierra Grande, localizada na comunidade de Hueyitlalpan no estado de Guerrero. Seu negócio é a apicultura, que se dedica à produção de colmeias para extração de mel e seus derivados como pólen, própolis e geleia real, que se transformam para gerar outros produtos desde a indústria alimentícia até produtos de higiene e higiene pessoal. Para analisar sua competitividade, foram realizadas entrevistas semiestruturadas; o primeiro a um dos sócios da empresa responsáveis pela planta de produção; oito entrevistas com residentes locais e uma com o comissário. Além disso, foram realizadas seis visitas para observar o processo de produção, distribuição e comercialização dos produtos. Em relação aos aspectos sociais e culturais das organizações rurais, foi realizada uma pesquisa bibliohemerográfica para estabelecer as bases teóricas. A empresa rural analisada possui um estilo particular de organização derivado das características socioculturais da população; É uma das empresas mais importantes da região central do estado. Fatores socioculturais como a religião, a relação com o meio ambiente, a população, as relações familiares e o conhecimento local influenciaram a construção de uma forma de organização na empresa rural, o que a torna capaz de ser competitiva. O conceito de competitividade aplicado às empresas rurais foi redefinido; aqui se trata da capacidade de um sistema organizado de indivíduos de gerar bens ou serviços de qualidade a custos mínimos, que sejam capazes de se manter no mercado (local, regional, nacional ou estrangeiro), a fim de obter lucro econômica e social.

Palavras-chave: competitividade social, cultura, empresa rural, identidade, território rural. Fecha Recepción: Noviembre 2019 Fecha Aceptación: Julio 2020 


\section{Revista Iberoamericana \\ de las Ciencias Sociales y \\ Humanísticas}

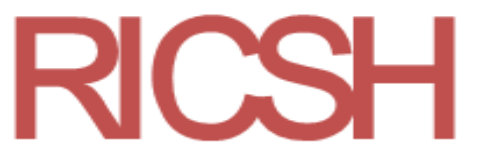

ISSN: $2395-7972$

\section{Introducción}

Por lo general, el término ruralidad ha estado relacionado con pobreza, rezago, marginación, y las actividades productivas realizadas en ese ámbito se vinculan a la agricultura y ganadería de subsistencia. Actualmente, el concepto en cuestión se ha sometido a un análisis que permite visualizar que los territorios rurales están siendo trastocados por el fenómeno de la globalización: las fronteras tanto culturales como geográficas se ven incesantemente modificadas por la conectividad tecnológica, lo que impacta los procesos sociales a través de los cuales los habitantes de las áreas rurales fincaban su cosmovisión de mundo.

Una de las principales características de las actividades económicas propias de un territorio rural, ya sean estas agrícolas, ganaderas, turísticas, artesanales, o las pequeñas empresas, entre otras, es su anclaje al territorio. Este anclaje territorial tiene como principal escala de análisis y de impacto socioeconómico lo local, y por lo mismo, el apego identitario de la población con su territorio al compartir una cultura, lo que influye en la construcción y reconstrucción de su espacio y en la revaloración de su entorno, además de fomentar la organización y participación de los actores locales en entidades de producción solidarias que tienen como fin generar un desarrollo comunitario y un conocimiento propio.

La diferencia entre la empresa rural y la empresa capitalista es que esta última se ha liberado de las limitaciones territoriales, sus accionistas toman decisiones a grandes distancias y pueden trasladar su empresa de un lugar a otro; de lo local solamente le interesa la explotación de los recursos naturales, la mano de obra barata o la captación del mercado para el consumo de sus productos; de esta manera, puede abandonar un territorio si así conviene a sus intereses, obligando a la localidad a ocuparse de los desechos, del impacto ambiental y de la reestructuración de la cohesión social (Bauman, 2001, p. 15).

Por otro lado, la empresa rural tiene como característica diferenciadora de la empresa capitalista su apego al territorio, recursos como el conocimiento y saberes locales, la familia como fuerza de trabajo; características que, para efectos de este trabajo, han sido englobadas como características socioculturales de la empresa. Con base en esto, se plantea la pregunta de si es posible que sean estas características las que influyen para que una empresa rural, en particular la empresa apicultora Miel Tierra Grande, sea exitosa y competitiva. 


\section{Revista Iberoamericana \\ de las Ciencias Sociales y \\ Humanísticas}

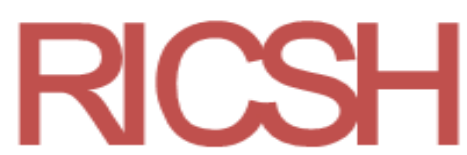

ISSN: 2395 - 7972

Cabe añadir que las empresas rurales, al ser unidades económicas de tipo social, no solo buscan el beneficio económico de sus miembros, sino que también tienen un fin social, que es el de contribuir al bienestar social como una de las formas para crear tejido empresarial en zonas rurales. Mozas y Bernal (2006), a partir del análisis de la economía social en áreas urbanas y rurales que realizan, arriban a la conclusión de que este tipo de empresas son agentes de desarrollo económico de la localidad donde se establecen, dado que "crean empleo, fijan a la población en su territorio, provocan un crecimiento empresarial, mejoran el bienestar social de la ciudadanía y favorecen las mejoras de las relaciones sociales, incentivan la cultura y la formación" (p. 137), entre otros beneficios. Sin embargo, pese a que las empresas sociales existen tanto en localidades urbanas como rurales, es en estas últimas donde se puede visualizar claramente la relación entre empresa rural y comunidad.

En este sentido, el objetivo general planteado en esta investigación es analizar cuáles son los aspectos socioculturales (cultura, identidad, saberes, relación familiar) que hacen a una empresa rural competitiva. Derivado de esta meta, se plantearon tres objetivos específicos: a) describir aspectos generales de la empresa rural (antecedentes, organización, modo de trabajo) para sustentar el por qué es competitiva; b) resaltar la importancia del conocimiento local, la cohesión social y la familia rural en la funcionalidad de una empresa rural, y c) analizar la importancia de la actividad que desempeña la empresa rural y la relación que tiene con el estilo de vida de las personas que la conforman. El logro de cada uno de los objetivos ayudó a responder la pregunta que se planteó al iniciar la investigación.

\section{Marco conceptual}

Como primer elemento de análisis, se describirá el concepto de territorio rural. Echeverri (11 de junio de 2007) lo define en los siguientes términos:

El territorio construido a partir del uso y apropiación de recursos naturales, donde se generan procesos productivos, culturales, sociales y políticos, nacidos del efecto de localización y apropiación territorial que se desprende de que los recursos naturales son factores de producción localizados (p. 1).

En esta definición se señala que las características del espacio territorial determinan las actividades productivas que en ese territorio se van a desarrollar. En las áreas rurales, la población tiene como unidad productiva la familia y su base de trabajo radica en la explotación y gestión de los recursos naturales. Existen dos elementos que son características 


\section{Revista Iberoamericana \\ de las Ciencias Sociales y \\ Humanísticas}

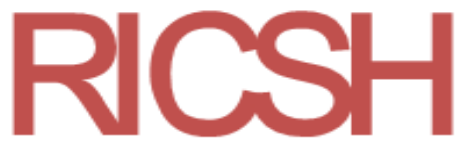

ISSN: $2395-7972$

fundamentales de la población rural. El primero de ello es la pertenencia a una comunidad local, donde la construcción de redes interpersonales es esencial no solo para manejar las estrategias económicas de las familias y sus miembros, sino que es importante para otros ámbitos de la vida cotidiana, como la amistad, la religión, el esparcimiento y el sentido de pertenencia. El segundo elemento es compartir un sistema sociocultural propio, en el que las creencias y normas complementan las relaciones y las instituciones sociales y viceversa.

La ruralidad no es exclusiva de las actividades agrícolas, sino que en esos territorios la población desempeña actividades varias como: agricultura, artesanía, pequeñas industrias, ganadería, actividades extractivas, turismo, entre otros. Lo rural trasciende lo agropecuario (Pérez, 2001), y mantiene una fuerte relación de intercambio con lo urbano: en el abastecimiento no solo de alimentos, sino también de otros bienes y servicios, entre los que se destacan la oferta y cuidado de los recursos naturales, los espacios para el descanso y los aportes al mantenimiento y desarrollo de la cultura.

\section{El territorio como espacio para la organización productiva}

Después de haber definido la categoría de territorio rural, se analizará el por qué una actividad como la organización productiva es una forma de uso del territorio. En primer lugar, a la apropiación del espacio se le denomina territorialización, y esta apropiación del territorio se manifiesta de distintas formas. Montañez (2001) señala que la territorialización es:

El grado de dominio que tiene determinado sujeto individual o social en cierto territorio o espacio geográfico, así como el conjunto de prácticas y sus expresiones materiales y simbólicas, capaces de garantizar la apropiación y permanencia de un territorio dado bajo determinado agente individual o social (p. 22).

Dentro de los sujetos que ejercen territorialidad, se encuentran los individuos, grupos sociales, grupos étnicos, empresas, el Estado nación, poderes fácticos, entre otros. De acuerdo con Gilberto Giménez (2005b), la apropiación del espacio puede ser prevalentemente utilitaria y funcional, o prevalentemente simbólico-cultural. Por ejemplo, cuando se considera al territorio como mercancía generadora de renta (valor de cambio), como fuente de recursos, como medio de subsistencia, como ámbito de jurisdicción del poder, como área geopolítica de control militar, como abrigo y zona de refugio, entre otras. Entonces, con una actividad como la apicultura, se está enfatizando el polo utilitario o 


\section{Revista Iberoamericana \\ de las Ciencias Sociales y \\ Humanísticas}

ISSN: $2395-7972$

funcional de la apropiación del espacio, ya que son las características físicas del territorio la fuente principal de la actividad que desempeñan.

El uso particular del territorio es la manifestación de la territorialidad. Esto se observa en los pequeños emprendimientos que surgen en esos espacios, la mayoría de ellos del sector primario. La agricultura se posiciona como la principal actividad que realiza la población de las zonas rurales, y la ganadería aparece en segundo lugar, ambas actividades de autoconsumo.

\section{Cultura e identidad en el espacio rural}

La identidad cultural tiene relevancia para comprender los procesos de organización de los actores locales en la construcción de su desarrollo. Según Guerra (1997), se trata de lo siguiente:

Un proceso que se construye en varios niveles de la praxis, en ritmos temporales distintos y en varias escalas espaciales (físicas, simbólicas, culturales, sociales) donde se dan cita, a su vez, diversas lógicas y tipos diferentes de relaciones sociales, siendo la representación que el sujeto tiene de todo ese proceso fundamental al mismo (p. 112).

Por lo tanto, la identidad cultural es de vital importancia para comprender los procesos de organización de los actores locales, en este caso los socios de la organización apícola, quienes han construido su estilo de organización con base en su cultura. Además, el concepto de identidad permite entender el porqué de sus acciones y cómo, desde su perspectiva, crean sus propias alternativas de desarrollo.

\section{Conocimiento local}

Se va a entender como conocimiento local al recurso del cual se vale la población rural para la realización de sus actividades cotidianas, incluyendo sus actividades económicas. Ya lo señalaba Echeverri (11 de junio de 2007) cuando hablaba de que se debe redefinir la economía del actual mundo rural:

Es menester incluir en las funciones de crecimiento y desarrollo aspectos, hasta hoy excluidos, como la economía ambiental y ecológica, los mercados de servicios ambientales, la sostenibilidad de los sistemas productivos, los recursos genéticos, los saberes y la cultura, como parte de una economía más 


\section{Revista Iberoamericana \\ de las Ciencias Sociales y \\ Humanísticas}

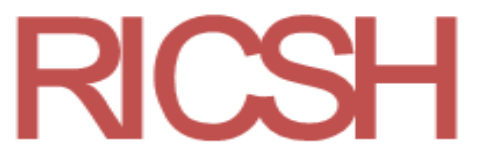

ISSN: $2395-7972$

compleja que la economía agraria que domina el escenario de política pública actual (p. 1).

De esta manera, las sociedades rurales tienen en común que su estrategia de sobrevivencia está basada en el uso múltiple de su espacio local, esto es, en su representación simbólica y cognitiva y en las formas de organización y apropiación de la naturaleza que rodea a dicho espacio (Barrera, 2015).

Por lo tanto, el conocimiento local significa una comprensión de lo local producto de la experiencia y observación de los agroecosistemas. Estos conocimientos son la base para la realización de cualquier actividad; sin embargo, la agricultura se ha tecnificado con el uso de máquinas para el arado, siembra y cosecha, y se han dejado de lado las prácticas agrícolas tradicionales, principalmente en los espacios donde hay cercanía con poblaciones urbanas.

El modo de trabajo de las unidades de producción rural posee características propias. Spicer (1971) pone énfasis en los elementos simbólicos que contribuyen a que algunos pueblos perduren, mientras que otros desaparecen. En este sentido, los valores, el vínculo con la tierra, con las semillas, con sus antepasados, con el lugar mismo, son elementos fundamentales de la persistencia campesina que configuran su identidad, la cual puede ser mucho más fuerte que cualquier racionalidad económica. Más que un modo de producción, la forma de organización campesina debe considerarse como un modo de vida (Mora, 2008, p. 126).

Como se puede observar, en estas definiciones se menciona que el uso de los saberes está principalmente relacionado con la población indígena, sin embargo, de acuerdo con diversos autores, el conocimiento local es utilizado por la población rural en general, porque es la base para realizar cualquier actividad.

El conocimiento local, de acuerdo con Farrington y Martin (1988), se considera como: "El acervo de conocimientos, creencias y costumbres consistentes entre sí y lógicos para quienes los comparten" (p. 23). Está constituido por saberes y percepciones únicos para una cultura o una sociedad dada (Grenier, 1998). Generalmente, deriva de observaciones cotidianas y de la experimentación con formas de vida, sistemas productivos y ecosistemas naturales. Estos conocimientos únicos son la ventaja que el habitante rural tiene para desempeñar de mejor manera sus actividades, ya que le permite buscar una solución a situaciones emergentes o desfavorables. 


\section{Revista Iberoamericana \\ de las Ciencias Sociales y \\ Humanísticas}

ISSN: $2395-7972$

La información que se obtiene sobre el conocimiento local es difícil de medir, así lo menciona Mora (2008), ya que la cantidad y calidad del conocimiento local sobre el entorno y la naturaleza varían entre los miembros de una comunidad, dependiendo de factores socioeconómicos, como género, edad, posición social, capacidad intelectual y profesión. Mora (2008) agrega que esta información es habitual que presente diversos grados de complejidad en una población determinada y varíe su nivel de consistencia entre quienes la poseen.

Por su parte, Nettin y Pimbert (citados en Mora, 2008) consideran que el saber que la población rural posee del entorno natural y de sus sistemas productivos les permite desenvolverse mejor bajo condiciones adversas, ecológicas o de mercado, y así lograr sus objetivos de producción.

\section{La empresa rural como elemento de desarrollo local}

Un proceso de desarrollo local supone una cultura de la proactividad en colectivo, que lleva a los actores locales a asumir riesgos, tomar la iniciativa, buscar alternativas, aprender de los errores, ser creativos y hacer que las cosas planeadas sucedan. Estas actitudes se convierten en condición para que todos los actores sociales se transformen en sujetos del desarrollo local. El desarrollo debe de venir desde dentro de las sociedades, es decir, de un esfuerzo endógeno, "movilizando las energías sociales que hacen que una sociedad se sienta responsable por sus acciones y por los resultados de ellas" (Cárdenas, 2002, p. 63).

Como ya se mencionó, las actividades agrícolas no son las únicas del sector rural, sino que se desempeñan diversas actividades, dependiendo de las características físicas y socioculturales de los territorios. El número de actividades que realiza la población de las zonas rurales va a garantizar que se dé un mayor o menor desarrollo. En sentido amplio, el desarrollo rural es:

Un proceso de mejora del nivel de bienestar de la población rural y de la contribución que el medio rural hace de forma general al bienestar de la población en su conjunto, ya sea urbana o rural, con su base de recursos naturales (Ceña, 1993, p. 32).

Los emprendimientos que surgen en el área rural son principalmente manejados por familias enteras. Desde la lógica campesina, son familias extensas las que se ocupan de una misma actividad productiva. Desde esta perspectiva, de acuerdo con Shanin (citado en 


\section{Revista Iberoamericana \\ de las Ciencias Sociales y \\ Humanísticas}

ISSN: 2395 - 7972

Schejtman, 1980), la unidad campesina es una "empresa de consumo-trabajo, con las necesidades de consumo de la familia como su objetivo y la fuerza de trabajo familiar como los medios, con un poco o ningún uso de trabajo asalariado" (p. 125). Aquí se considera que las actividades productivas están arraigadas a una cultura e identidad de la población rural, que es parte del día a día y que esa es razón suficiente para perdurar.

Por otro lado, la empresa rural tiene una doble finalidad: la económica y la social. En lo económico, genera utilidades, remunera al trabajo y produce bienes y servicios que satisfacen las necesidades del consumidor; y en lo social, contribuye al desarrollo integral de socios y trabajadores, y ayuda a la obtención del bien común (Secretaría de Agricultura, Ganadería, Desarrollo Rural, Pesca y Alimentación [Sagarpa], 2006). En la empresa apícola objeto de estudio, el negocio fue dejando ganancias a los socios. Uno de ellos lo expresa así: "Mis hermanos mayores y mi papá se inclinaron en la inversión en la apicultura, era más rentable trabajar con las abejas" (Morales, 14 de abril de 2016, entrevista personal). Es decir, se dieron cuenta de que en la apicultura no tenían que trabajar todos los días y eran mínimas las actividades y el tiempo empleado, por lo que decidieron que ese sería el negocio familiar.

Con base en lo analizado, se considera que la empresa rural es un sistema organizado socioculturalmente que tiene como una de sus funciones la de ofrecer un servicio o, con un mínimo de tecnología, producir o transformar los recursos naturales en bienes de consumo con fines lucrativos. Y se diferencia de la empresa con localización urbana por el hecho de que cumple una función importante en la sociedad, que es la de buscar el bienestar colectivo sin dejar de lado la identidad con el territorio, lo que también la fortalece y le permite una mayor sustentabilidad.

Así, la empresa rural tiene como ventaja el potencial de la mano de obra, considerando que son entidades donde la estructura humana de trabajo se basa en la confianza y relaciones de autoridad definidas, lo que es clave para hacerla competitiva. La fuerza de trabajo proviene de la familia misma, y no dispone de una retribución fija para ninguno de sus miembros, por lo que se determina que el ingreso sea variable y ligado estrechamente a resultados.

Una de las características de las empresas rurales es que la familia es la base de la mayoría de los emprendimientos. El apoyo de sus miembros, principalmente de quienes dirigen la empresa, es hasta cierto punto desinteresado, honesto y franco porque responde, no solo al interés económico, sino también al afectivo, producto de la relación consanguínea. 


\section{Revista Iberoamericana \\ de las Ciencias Sociales y \\ Humanísticas}

ISSN: $2395-7972$

De acuerdo con Walters, Cintrón y Serrano (2006), la familia es "una de las instituciones sociales que influye, regula, canaliza e impone significado social y cultural en la vida de las personas" (p. 18). Palacios y Rodrigo (citados en Herrera, Weisser y Salazar, 2004), por su parte, definen a la familia de la siguiente forma:

La unión de personas que comparten un proyecto vital de existencia en común que se quiere duradero, en el que se generan fuertes sentimientos de pertenencia a dicho grupo, existe un compromiso personal entre sus miembros y se establecen intensas relaciones de intimidad, reciprocidad y dependencia" (p. 8).

Del involucramiento de la familia en el trabajo de la empresa, se desprenden algunas otras características que son propias de este tipo de organización, tal es el caso del capital social, producto de las relaciones afectivas que existen entre los miembros de quienes conforman el grupo familiar. Al respecto, Durston (2002) define el capital social como el "contenido de ciertas relaciones y estructuras sociales, es decir, actitudes de confianza que se dan en combinación con conductas de reciprocidad y cooperación" (p. 16). Mientras que para Bourdieu (1986) el capital social es la suma de los recursos reales o potenciales ligados a la posesión de una red duradera de relaciones de reconocimiento mutuo más o menos institucionalizadas. Coleman (1988) sugiere algo similar, y agrega que al ser un capital más contribuye a alcanzar los logros fijados, y que es tan importante como el capital financiero, humano y natural. El capital social está formado por características como el apoyo entre los miembros, la confianza, solidaridad, honestidad, ahorro, trabajo equitativo, respeto, constancia, continuidad, comunicación, entre otros. Todos estos valores son producto de los lazos de parentesco y cuyo objetivo común es lograr el bienestar familiar.

Por lo tanto, en las sociedades rurales, desde la perspectiva de la economía campesina, los procesos productivos son desarrollados por unidades de tipo familiar, lo que asegura, ciclo a ciclo, que se reproduzcan sus condiciones de vida y de trabajo. Y garantiza, en primer término, los medios de sostenimiento biológico y cultural de los miembros de la familia; y en segundo término, destina un fondo para la reposición de los medios de producción para cubrir las eventualidades que puedan poner en riesgo al grupo familiar: enfermedades, gastos ceremoniales, entre otras (Schejtman, 1980). 


\section{Revista Iberoamericana \\ de las Ciencias Sociales y \\ Humanísticas}

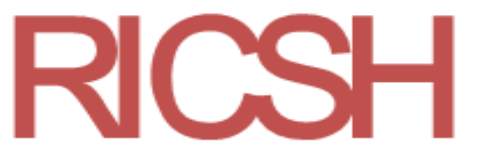

ISSN: $2395-7972$

\section{La competitividad de la empresa rural}

Con el avance de la globalización nuevas formas de hacer economía en las zonas rurales van surgiendo. Esto a pesar de que el concepto de competitividad parecería no tener cabida en la ruralidad, ya que tiene un origen puramente capitalista, como se desprende de la definición del Canada. Task Force on Competitiveness in the Agri-food Sector, citado por Solleiro y Castañón (2004), quienes, por cierto, definen la competitividad como la capacidad permanente para ganar y sostener una participación rentable en el mercado. Sin embargo, en la nueva ruralidad se han adoptado estrategias productivas ajenas a lo tradicional, y una de estas es que la pequeña empresa rural puede llegar a ser competitiva.

De esta manera, el reto que tienen las empresas rurales es el de volverse competitivas para cumplir satisfactoriamente las necesidades económicas de la familia o familias que las conforman, para garantizar su desarrollo y consolidación, sin dejar de lado la importante función social que tienen: mejorar la calidad de vida de las familias y habitantes de la localidad a través de su incidencia en el desarrollo local.

La competitividad de la empresa depende de factores internos y externos. Los factores internos son aquellos que condicionan su capacidad de crear valor; en tanto que los factores externos provienen del entorno (factores macroeconómicos, institucionales o de estructura de mercado), los cuales condicionan la competitividad de las empresas dentro de una región (Montes, Fernández y Vázquez, 2012).

Para que el término competitividad funcione en la ruralidad, se debe tener presente la diferencia de la empresa rural con la empresa capitalista que se relaciona con la retribución social que genera a la localidad en donde está inserta. Igualmente, para redefinir la competitividad en el espacio rural, se debe considerar el concepto de beneficio social que del funcionamiento de la empresa rural se desprende:

Cuando se incorporan las externalidades, es decir, los beneficios sociales de las actividades productivas rurales, es posible incorporar el concepto de competitividad social, como expresión de una economía de eficiencia que dé cuenta cabal de las retribuciones que esta tiene para el conjunto social, incluyendo a los empresarios y a las rentabilidades privadas, pero no limitando su valoración a este ámbito (Echeverri, 2007, p. 2). 


\section{Revista Iberoamericana \\ de las Ciencias Sociales y \\ Humanísticas}

ISSN: $2395-7972$

Con el término de competitividad social se incluyen los aspectos de eficiencia productiva sin limitarse a la rentabilidad, sino ajustándolo a lo que es actualmente la economía rural. Una definición propia a partir de las ya analizadas sería la siguiente: la competitividad es la capacidad que tiene un sistema organizado de individuos para generar bienes o servicios de calidad, a costos mínimos, que estén en posibilidad de consumirse en el mercado (local, regional, nacional o extranjero), con la finalidad de lograr un beneficio económico y social.

\section{Método}

En presente investigación de tipo analítico-exploratorio se utilizó una metodología cualitativa. Primero se buscó información documental sobre estudios de casos similares, y posteriormente se consultaron páginas de Internet de dependencias como el Instituto Nacional de Estadística, Geografía e Informática (Inegi), el Consejo Nacional de Evaluación de la Política de Desarrollo Social (Coneval), el Consejo Nacional de Población (Conapo), la Sagarpa y la Secretaría de Desarrollo Social (Sedesol), con el fin de tener mayor información sobre el contexto de la localidad a través de datos generales socioeconómicos, estadísticas y mapas.

El trabajo de campo tuvo dos fases. La primera consistió en la realización de recorridos en la comunidad para la observación, con el fin de analizar el entorno de los habitantes de la localidad y de la empresa apícola. En la segunda fase se realizaron entrevistas semiestructuradas; la primera de ellas se realizó a uno de los socios de la empresa apícola y encargado de la planta productora, luego se llevaron a cabo ocho entrevistas más a habitantes de la localidad de Hueyitlalpan. También se tuvo una entrevista con el ahora excomisario para conocer aspectos históricos y datos generales de los habitantes de dicha comunidad.

Hueyitlalpan, en contraste con su significado en náhuatl, que es 'tierra grande', es un territorio pequeño que se ubica en la zona donde se asentó la cultura mexica en el estado de Guerrero; es la entrada al municipio de Mártir de Cuilapan viniendo desde Almolonga; está rodeado de grandes elevaciones que se desprenden de la Sierra Madre del Sur, lo que lo hace un lugar tranquilo, de clima agradable, fresco y tierra fértil, que permite el principal sustento de quienes la habitan: la agricultura. 


\section{Revista Iberoamericana \\ de las Ciencias Sociales y \\ Humanísticas}

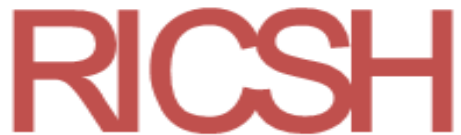

ISSN: $2395-7972$

Hueyitlalpan es, después de Apango, la cabecera municipal, una de las más importantes localidades del municipio de Mártir de Cuilapan, por su cercanía con manantiales que abastecen del vital líquido a seis de las localidades del territorio municipal. En el siguiente mapa se muestra la ubicación de la localidad donde está establecido el objeto de estudio. Además, se visualiza la cercanía que tiene con la ciudad de Apango, cabecera municipal del municipio.

Figura 1. Ubicación de la localidad de Hueyitlalpan

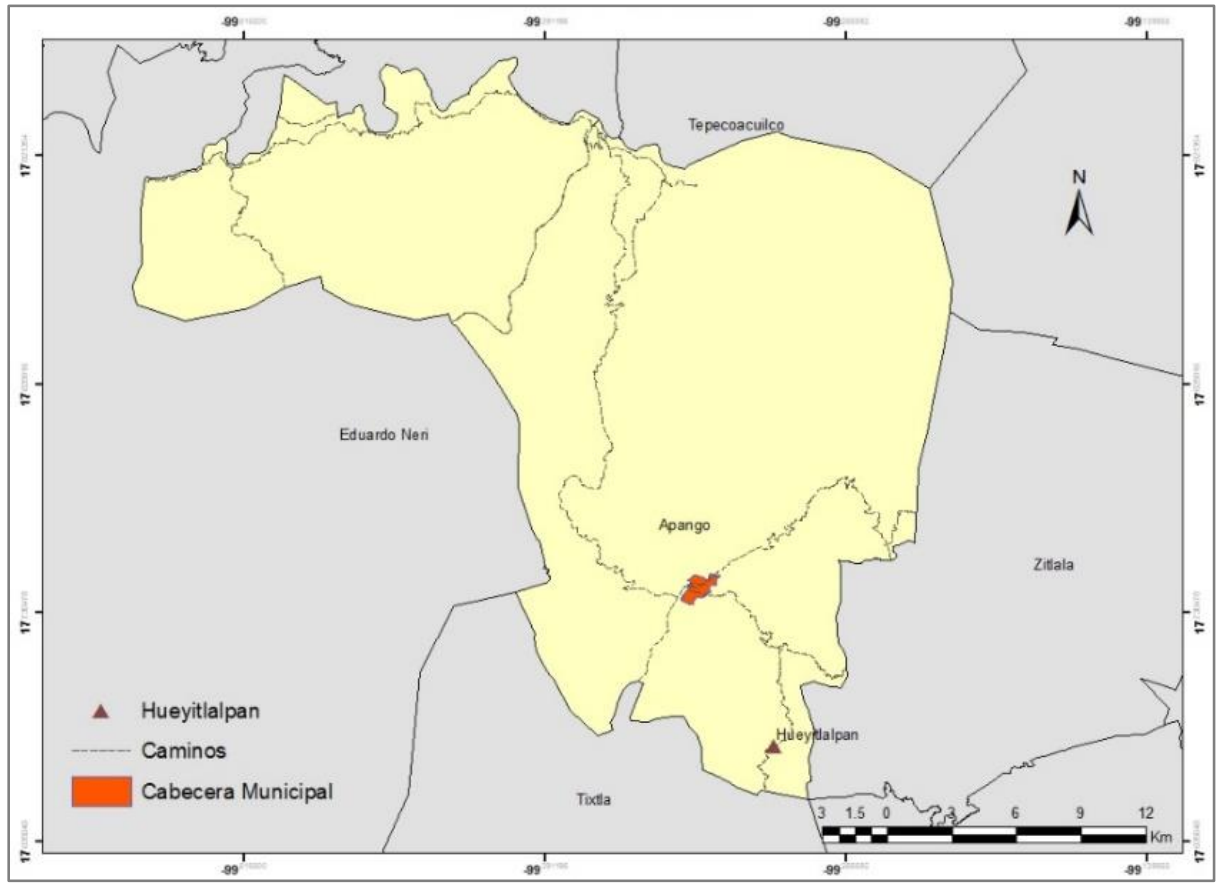

Fuente: Elaboración propia con base en Inegi (2009)

\section{Resultados}

La empresa apícola Miel Tierra Grande tiene una composición familiar y sus miembros son de la misma localidad; esto quiere decir que no solamente comparten lazos familiares, sino también identidad y cultura, lo que les permite trabajar cohesionados y trasladar los valores culturales y de organización familiar a la empresa. Por ejemplo, al ser una familia rural, comparten valores como la solidaridad, cooperación, comunicación y respeto, aunque mantienen una organización jerárquica. 


\section{Revista Iberoamericana \\ de las Ciencias Sociales y \\ Humanísticas}

ISSN: $2395-7972$

Otro de los aspectos observados fue que la toma de decisiones está guiada por una doctrina conductual de tipo religioso. En este caso, de la religión evangélica, la cual tiene entre sus principios el valor al trabajo, el sacrificio, el ahorro y la relación familiar armoniosa. Estas características de tipo cultural son las que guían su toma de decisiones.

Por otro lado, su relación con las abejas va más allá de verlas solo como productoras de miel, sino que han incorporado al bagaje cultural de la familia parte de su comportamiento, como es el trabajo colectivo, la distribución de las actividades, la jerarquización en la toma de decisiones, entre otras.

Otra característica que influye en la competitividad de la empresa es el giro. Al ser la apicultura una actividad sustentable, preserva el entorno natural, produce beneficio económico entre quienes llevan a cabo la actividad y un beneficio social al mejorar las condiciones de vida de los integrantes de la familia y de los habitantes de la localidad. Con esta actividad, los socios de la apícola no solo venden miel, sino que también venden una diversidad de productos derivados de esta.

Los saberes locales provenientes de la relación con el territorio es otra característica que han utilizado en el trabajo de la empresa. Aunado a ello, se han apoyado del conocimiento sistematizado y de la tecnología para corregir procesos, adoptar maquinaria, equipo y técnicas en sus labores, gracias a lo cual han mejorado su eficiencia en la producción y enriquecido su bagaje cultural al construir conocimiento nuevo que responde a sus intereses contextuales y como colectivo.

También se observó que, aunque no poseen estudios profesionales sobre administración de empresas, sobre la marcha se han ido capacitando, ya que han implementado estrategias de organización para la toma de decisiones, lo que les ha permitido volverse autosuficientes y posicionarse como una empresa importante en la región.

Como parte de la organización de las actividades productivas del grupo familiar, a continuación, se describe la división del trabajo:

- $\quad$ Apicultura. Se trata del principal trabajo del grupo familiar. La miel es el principal producto extraído de la colmena para su posterior procesamiento, envasado y comercialización.

- $\quad$ Taller de carpintería. En el taller se elaboran los cajones que necesitan para instalar las colmenas, pero también elaboran cajones para su venta al público. 


\section{Revista Iberoamericana \\ de las Ciencias Sociales y \\ Humanísticas}

ISSN: 2395 - 7972

- Vivero de plantas forestales. El vivero tiene estrecha relación con la primera fase de la apicultura, tiene la función de producir plantas de la región con la finalidad de sembrarlas en las localidades donde se encuentran las colmenas, con el fin de que las abejas siempre tengan al alcance el néctar que se necesita para producir miel.

- $\quad$ Construcción. Esta área es la que se encarga de realizar todas las actividades de construcción, ampliación y remodelación de los espacios de la empresa, así como de las viviendas de los integrantes del grupo familiar.

- $\quad$ Agricultura y ganadería. Estas actividades son parte de la vida de todos los habitantes de la zona; para el grupo familiar son actividades que desde generaciones atrás han estado realizando.

Las áreas en las que se desempeña el grupo familiar fueron surgiendo por el interés de ser autosuficientes, es decir, de depender de su propio trabajo, además de que utilizaron parte de los conocimientos que en la localidad habían aprendido, como la carpintería o la construcción.

Más que un deber, el trabajo con la apicultura es parte de la vida de quienes comenzaron ese proyecto. Existe un vínculo que sobrepasa el de ser solo el medio de subsistencia familiar. En este vínculo se encuentran involucrados sentimientos, vivencias, sacrificios y el esfuerzo que han puesto para mantener operando el negocio. También, los ahora socios, han crecido junto con la empresa. Aunque su trabajo principal era la agricultura, por necesidad y para aprovechar la oportunidad que se les presentó, tuvieron que estar abiertos a aprender sobre la cultura del manejo de las abejas, las que, a partir de ese momento, los han acompañado por más de veinte años.

El señor Moisés relata que la población ya estaba acostumbrada a convivir con las abejas antes de su africanización, pero ellos tuvieron que adaptarse a las dificultades que provocó la abeja africana y a especializarse a fondo en la apicultura. La familia rural posee una cultura e identidad más estrecha con el territorio, además de que la unión familiar se encuentra más valorizada, al igual que el trabajo colectivo; sin embargo, también se ven influenciados por el proceso de urbanización y de los avances tecnológicos, por lo que el trabajo apícola que hoy realizan dista mucho del que realizaban hace quince años; han tecnificados sus procesos para mejorar la calidad y estar en condiciones para competir a nivel nacional e internacional. 


\section{Revista Iberoamericana \\ de las Ciencias Sociales y \\ Humanísticas}

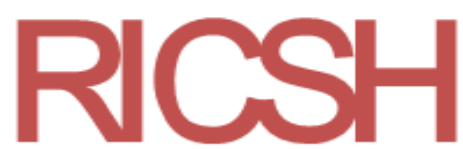

ISSN: 2395 - 7972

Aunque han tecnificado su proceso de trabajo, no han abandonado las enseñanzas que las abejas les mostraron. Así lo cuenta don Moisés, uno de los socios y encargado de la planta de producción:

Una abeja tiene varios trabajos. Nace y su primer trabajo es hacer limpieza en las celdas reales y alimentar a las larvas, ese es su primer trabajo. No cuestiona, si no quiere, y si no trabaja: la matan; si nace defectuosa, si le falta un ala, si le falta una pata: la matan. No hay incapacitados dentro de una colmena, suena duro pero es una realidad (Morales, 14 de abril de 2016, entrevista personal).

El señor Moisés también relató la importancia del trabajo para las abejas. La abeja vive su ciclo trabajando, y en gran medida el núcleo familiar se identifica con el trabajo de estos pequeños insectos:

Después de eso empieza a hacer trabajos de guardián en la colmena, por seguridad de ellas, si ve un objeto que se mueve, va, lo identifica y si se pone agresivo da la alarma y son las primeras que van a atacar. Cuando ya es una abeja adulta, es la que sale al campo y se va por néctar, muere en el campo, porque por lo regular no se da mortandad en las colmenas, mucha abeja adulta muere en el campo y muere en el trabajo, trabajando (Morales, 14 de abril de 2016, entrevista personal).

Este conocimiento sobre el trabajo de las abejas es una característica que como apicultores adoptan, complementa sus valores culturales y fortalece su identidad; ya no es solo el conocimiento que tenían cuando se desempeñaban solo como agricultores, sino que ahora han conjuntado el conocimiento sobre el ciclo de vida de las abejas en la recreación de su identidad.

\section{Discusión}

En el transcurso de la investigación se presentaron algunas dificultades derivadas del problema de inseguridad que se vive en el estado de Guerrero, particularmente en el tramo carretero de Chilpancingo a Tixtla, siendo ésta la vía de acceso al municipio de Mártir de Cuilapan; la situación sociopolítica del municipio también generó bloqueos carreteros en las principales vías de acceso al mismo, producto de inconformidades de la población con las autoridades del ayuntamiento. Estos hechos impidieron que se realizara en varias ocasiones 


\section{Revista Iberoamericana \\ de las Ciencias Sociales y \\ Humanísticas}

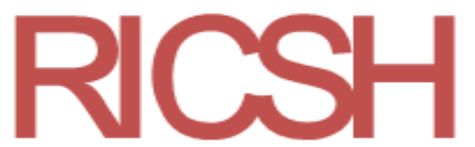

ISSN: 2395 - 7972

el trabajo de campo trazado en la localidad de Hueyitlalpan. Sin embargo, la fortaleza de la investigación radica en el estudio que se hace de una empresa rural que lleva varios años en el ramo de la apicultura y que mantiene su estructura familiar como base de su organización.

Al finalizar el estudio y análisis del caso de la empresa apicultora Miel Tierra Grande, se identificó que debido a su tamaño en cuanto a producción y su impacto en la región centro del estado de Guerrero, es una empresa competitiva económica y socialmente, dado que satisface parte de la demanda del mercado local y exporta al extranjero a través de un intermediario, y las características de tipo sociocultural que se identificaron fueron el tipo de religión que profesan, la solidez de las relaciones familiares y el conocimiento de su entorno, las cual influyen en construcción de esa competitividad.

Con la actual constitución de la empresa familiar, se tiene al frente de la organización a los hermanos quienes han dividido el trabajo por áreas, siendo la principal la apicultura, después están: el taller de carpintería, donde se fabrican las colmenas y algunas otras herramientas; el vivero de plantas forestales, ganadería y finalmente el área de agricultura.

\section{Conclusiones}

La religión se considera como el elemento más importante en la construcción de su estilo de trabajo, organización y de vida, es una forma de expresión cultural y se exterioriza en la conducta y forma de pensar de las personas.

La religión como una expresión cultural marca las actividades que realizan quienes la profesan, ya que tiene influencia sobre el comportamiento de los individuos en la vida cotidiana, inclusive en el aspecto económico, como es el caso de los evangélicos, ${ }^{1}$ quienes son fieles a lo que está escrito en la Biblia, como lo señala Weber (1994) en su obra La ética protestante y el espíritu del capitalismo; los protestantes ${ }^{2}$ valoran mucho el trabajo, el sacrificio, el ahorro, la familia y el hacer riqueza en el nombre de Dios. A diferencia de los católicos, quienes son conformistas y prefieren la seguridad, los evangélicos son propensos a enfrentar riesgos; mientras los católicos ponen énfasis en la confesión de sus pecados para su posterior perdón, los protestantes se enfocan en la conducta: trabajo, honestidad, no alcohol, no fiestas, sí familia, sí ahorro.

\footnotetext{
${ }^{1}$ Se hace hincapié en la religión evangélica porque es la religión que practica la familia que dirige la organización apicultora.

${ }^{2}$ Weber hace referencia a las religiones que se separaron del catolicismo, y pone especial énfasis al calvinismo ya que lo consideraba más influyente para el modo de vida de la clase burguesa.
} 


\section{Revista Iberoamericana \\ de las Ciencias Sociales y \\ Humanísticas}

ISSN: $2395-7972$

La Biblia ha sido una guía para toda la familia, les ha servido para poder realizar el trabajo dentro de la organización. Esta es una razón de peso por la que el grupo familiar dedica mucho tiempo al trabajo, de quienes profesan la misma fe los orienta para el trabajo del grupo. El grupo familiar está convencido de que si no acataran esta premisa no habrían logrado lo que son ahora como empresa.

Actualmente, la empresa familiar cuenta con la certificación en Buenas Prácticas de Producción, así como en Buenas Prácticas de Manejo y Envasado de la Miel y la certificación de Criadero de Abejas Reinas y Núcleos de Abejas. Gracias a esta última ya no solo crían para su propio uso, sino que venden abejas reina, abejas y núcleos, ${ }^{3}$ así como cajones para las colmenas al público en general. Estas certificaciones les permitirán hacer negocios con cadenas de distribución más grandes y también les permitirán exportar su miel con su propia marca.

Posterior a haber invertido en su producto estrella, la miel, desde hace tres años a la fecha han estado trabajando en la producción a gran escala de polen, segundo producto que más ganancias les genera, con fines de exportación a través de un intermediario que se encuentra en el estado de Querétaro. En el mismo ramo de la apicultura, incursionaron en la venta de equipo para el manejo de las abejas, como guantes, ahumadores, velos, trajes, entre otros, aunque no son productores de estos materiales, ellos los ponen al alcance de sus clientes.

Después de haber analizado la empresa apícola, se puede concluir que es una empresa competitiva tanto económica como socialmente, y que factores como la cultura, identidad, saberes y relación familiar han influido en la forma en la que se han organizado, la cual ha sido clave para posicionarse como una de las empresas rurales más importantes de la región, no solo por las ventas obtenidas en lo local, sino por su trascendencia a lo nacional e internacional.

${ }^{3}$ Los núcleos consisten en una pequeña familia de abejas que puede estar formado por cuatro o cinco marcos. 


\section{Revista Iberoamericana \\ de las Ciencias Sociales y \\ Humanísticas}

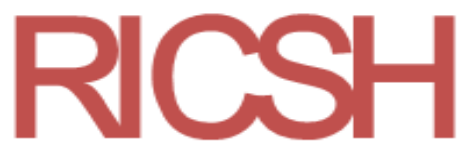

ISSN: $2395-7972$

\section{Referencias}

Barrera, N. (2015). Saberes locales y geo-metrías del paisaje: un giro interdisciplinario desde la alteridad. En Vázquez, A. (ed.), Las geometrías de la desigualdad. Modernidad, globalidad y poder (pp. 173-233). Querétaro, México: Universidad Autónoma de Querétaro.

Bauman, Z. (2001). La globalización, consecuencias humanas. México: FCE.

Bourdieu, P. (1986). The forms of capital. In Richardson, J. G. (ed.), Handbook of theory and research for the sociology of education. Westport, United States: Greenwood.

Cárdenas, N. (2002). El desarrollo local su conceptualización y procesos. Provincia, (8), 5376. Recuperado de https://www.redalyc.org/pdf/555/55500804.pdf.

Ceña, F. (1993). El desarrollo rural en sentido amplio. En Ramos, E. y Caldentey, P. (eds.), El desarrollo rural andaluz a las puertas del siglo XXI (pp. 25-40). Sevilla, España: Consejería de Agricultura y Pesca.

Coleman, J. S. (1988). Social Capital in the Creation of Human Capital. American Journal of Sociology, 94, 95-120.

Consejo Nacional de Evaluación de la Política de Desarrollo Social [Coneval]. (2016). Resultados de pobreza en México 2016 a nivel nacional y por entidades federativas. México: Consejo Nacional de Evaluación de la Política de Desarrollo Social. Recuperado de http://www.coneval.org.mx/Medicion/MP/Paginas/Pobreza_2016.aspx.

Cordero, P., Chavarría, H., Echeverri, R. y Sepúlveda, S. (2003). Territorios rurales, competitividad y desarrollo. San José, Costa Rica: IIC.

Courlert, C. et Pecqueur, B. (1994). Districts industriels, systèmes productifs, localisés et développement. In Benko, G. et Lipietz, A. (eds.), Les régions qui gagnent; districts et réseaux, les nouveaux paradigmes de la géographie économiques (pp. 81-102). Grenoble, France: IREP.

Delegación Sader Hidalgo. (30 de agosto de 2013). Darle un valor agregado a la producción de miel es el reto de la apicultura en el país. Blog de la Delegación Sader Hidalgo. Recuperado de https://www.gob.mx/sagarpa/hidalgo/articulos/darle-un-valoragregado-a-la-produccion-de-miel-es-el-reto-de-la-apicultura-en-el-pais?idiom=es.

Durston, J. (2002). El capital social campesino en la gestión del desarrollo rural. Díadas, equipos, puentes y escaleras. Santiago, Chile: Naciones Unidas: Cepal. 


\section{Revista Iberoamericana de las Ciencias Sociales y Humanísticas}

ISSN: $2395-7972$

Echeverri, R. (11 de junio de 2007). Economía y competitividad del territorio rural. agua.org.mx. Recuperado de https://agua.org.mx/biblioteca/economia-ycompetitividad-del-territorio-rural/.

Farrington, J. and Martin, A. (1988). Farmer Participation in Agricultural Research: A Review of Concepts and Practices. London, England: Overseas Development Institute.

Fernández, F. (2006). Geografía cultural. En Hiernaux D. y Lindón, A. (dirs.), Tratado de geografía humana (pp. 220-253). Ciudad de México, México: Anthropos.

Giménez, G. (2005b). Territorio, paisaje y apego socio-territorial. En Antología sobre culturas populares e indígenas (pp. 316-329). México: Conaculta.

Gómez, S. (2002). Una nueva ruralidad. ¿Qué tan nueva? Chile: Universidad Austral de Chile, Ediciones LOM.

González, F. (2012). Territorio, cultura e interculturalidad. Una mirada a educación indígena en Guerrero. México: Universidad Autónoma de Guerrero.

Grenier, L. (1998). Working with Indigenous Knowledge: A Guide for Researchers. Ottawa, Canada: IDRC.

Guerra, C. (1997). Hacia una sociología del sujeto: democracia y sociedad civil. En León, E. y Zemelman, H. (coords.), Subjetividad: umbrales del pensamiento social (pp. 107136). México: Anthropos.

Herrera, B., Weisser, B. y Salazar, D. (2004). Imágenes y conceptos de familia expresados en las representaciones sociales de mujeres de sectores urbano - populares de la ciudad de Temuco, Chile. Límite, 1(11), 1-34.

Instituto Nacional de Geografía y Estadística [Inegi]. (2009). Prontuario de información geográfica municipal de los Estados Unidos Mexicanos. México: Instituto Nacional de Geografía y $\quad$ Estadística. Recuperado de http://www3.inegi.org.mx/contenidos/app/mexicocifras/datos_geograficos/12/12042 .pdf.

Montañez, G. (2001). Razón y Pasión del Espacio y el Territorio. En Espacio y territorio. Razón pasión e imaginarios (pp. 15-32). Bogotá, Colombia: Unibiblios.

Montes, J., Fernández, E. y Vázquez, C. (2012). La competitividad de la empresa. Un enfoque basado en la teoría de recursos. Oviedo, España: Universidad de Oviedo. 


\section{Revista Iberoamericana \\ de las Ciencias Sociales y \\ Humanísticas}

ISSN: $2395-7972$

Mora, J. (2008). Persistencia, conocimiento local y estrategias de vida en sociedades campesinas. Revista de Estudios Sociales, (29), 122-133.

Mozas, A. y Bernal, E. (2006). Desarrollo territorial y economía social. Revista de Economía Pública, Social y Cooperativa, (55), 125-140. Recuperado de https://www.redalyc.org/pdf/174/17405505.pdf.

Pérez, E. (2001). Repensando el desarrollo rural. En Giarracca, N. (comp. ${ }^{\text {a) }}$ ¿Una nиeva ruralidad en América Latina? (pp. 17-30). Buenos Aires, Argentina: CLACSO.

Schejtman, A. (1980). Economía campesina: Lógica interna, articulación y persistencia. Revista de la CEPAL, (11), 121-140.

Secretaría de Agricultura, Ganadería, Desarrollo Rural, Pesca y Alimentación [Sagarpa]. (2006). La empresa rural y las redes empresariales. México: Secretaría de Agricultura, Ganadería, Desarrollo Rural, Pesca y Alimentación.

Secretaría de Desarrollo Social [Sedesol]. (2013). Catálogo de localidades. México: la Secretaría de Desarrollo Social. Recuperado de http://www.microrregiones.gob.mx/catloc/LocdeMun.aspx?tipo=clave\&campo=loc \&ent=12\&mun=042.

Secretaría de Desarrollo Social [Sedesol]-Consejo Nacional de Evaluación de la Política de Desarrollo Social [Coneval]. (2010). Informe anual sobre la situación de pobreza y rezago social. Mártir de Cuilapan. Ciudad de México, México: Secretaría de Desarrollo Social-Consejo Nacional de Evaluación de la Política de Desarrollo Social.

Solleiro, J. y Castañón, R. (2004). Competitividad y sistemas de innovación: los retos para la inserción de México en el contexto global. En Globalización, ciencia y tecnología. Volumen II (pp. 165-197). Bogotá, Colombia: Organización de Estados Iberoamericanos para la Educación, la Ciencia y la Cultura-Corporación Escenarios. Spicer, E. H. (1971). Persistent cultural systems. Science, 174(4011), 795-800.

Walters, K., Cintrón, F. y Serrano, I. (2006). Familia reconstituida. El significado de "familia" en la familia reconstituida. Psicología Iberoamericana, 14(2), 16-27.

Weber, M. (1994). La ética protestante y el espíritu del capitalismo. Ciudad de México, México: Ediciones Coyoacán. 


\section{Revista Iberoamericana de las Ciencias Sociales y Humanísticas}

ISSN: $2395-7972$

\begin{tabular}{|c|c|}
\hline Rol de Contribución & Autor (es) \\
\hline Conceptualización & $\begin{array}{l}\text { Indira Flores Salgado (principal), Floriberto González } \\
\text { González (apoyo) }\end{array}$ \\
\hline Metodología & $\begin{array}{l}\text { Indira Flores Salgado (igual), Floriberto González González } \\
\text { (igual) }\end{array}$ \\
\hline Software & NO APLICA \\
\hline Validación & $\begin{array}{l}\text { Floriberto González González (igual), Indira Flores Salgado } \\
\text { (igual) }\end{array}$ \\
\hline Análisis Formal & $\begin{array}{l}\text { Indira Flores Salgado (principal), Floriberto González } \\
\text { González (apoyo) }\end{array}$ \\
\hline Investigación & $\begin{array}{l}\text { Indira Flores Salgado (principal), Floriberto González } \\
\text { González (apoyo) }\end{array}$ \\
\hline Recursos & Indira Flores Salgado (principal) \\
\hline Curación de datos & NO APLICA \\
\hline $\begin{array}{l}\text { Escritura - Preparación del } \\
\text { borrador original }\end{array}$ & $\begin{array}{l}\text { Indira Flores Salgado (principal), Floriberto González } \\
\text { González (apoyo) }\end{array}$ \\
\hline $\begin{array}{l}\text { Escritura - Revisión y } \\
\text { edición }\end{array}$ & $\begin{array}{l}\text { Floriberto González González (igual), Indira Flores Salgado } \\
\text { (igual) }\end{array}$ \\
\hline Visualización & $\begin{array}{l}\text { Indira Flores Salgado (principal), Floriberto González } \\
\text { González (apoyo) }\end{array}$ \\
\hline Supervisión & $\begin{array}{l}\text { Floriberto González González (principal), Pedro Vidal Tello } \\
\text { Almaguer (apoyo) }\end{array}$ \\
\hline $\begin{array}{l}\text { Administración de } \\
\text { Proyectos }\end{array}$ & Indira Flores Salgado (principal) \\
\hline Adquisición de fondos & $\begin{array}{l}\text { Floriberto González González (principal), Pedro Vidal Tello } \\
\text { Almaguer (apoyo) }\end{array}$ \\
\hline
\end{tabular}

